\title{
Comparison of Chronic Angioscopic Findings of Bare Metal Stents, 1st-Generation Drug-Eluting Stents and 2nd-Generation Drug-Eluting Stents

\author{
- Multicenter Study of Intra-Coronary Angioscopy \\ After Stent (MICASA) -
}

Kazuoki Dai, MD; Hiroshi Matsuoka, MD, PhD; Hideo Kawakami, MD, PhD; Tetsuya Sato, MD, PhD; Kouki Watanabe, MD, PhD; Yasuharu Nakama, MD; Masaharu Ishihara, MD, PhD

\begin{abstract}
Background: No previous study has reported a comprehensive comparison of the chronic angioscopic findings after bare metal stent (BMS), and 1st- and 2nd-generation drug-eluting stents (DES).

Methods and Results: The Multicenter Study on Intra-Coronary Angioscopy after Stent (MICASA) is a multicenter registry of coronary angioscopy. A total of 264 stents were observed by coronary angioscopy 1 year after PCl. There were $15 \mathrm{BMS}, 90$ 1st-generation DES, and 159 2nd-generation DES. Neointimal coverage (NC) of the stent was classified into 4 grades from 0 (no coverage) to 3 (complete coverage). Yellow color (YC) of plaque at the stented segment was graded from 0 (white) to 3 (bright yellow). Minimum (Min-) and Maximum (Max-) NC grade were significantly lower with 1st- and 2nd-generation DES than with BMS. Although the Max-NC grade was similar, the Min-NC grade was significantly higher for 2nd-generation DES than for 1st-generation DES. Both the YC grade and the incidence of thrombus with 2nd-generation DES were lower than with the 1st-generation DES and were comparable to BMS. Multivariate analysis showed that low-density lipoprotein, 1st-generation DES, and acute coronary syndrome were independent factors for yellow plaque (YG2 or 3), and that hypertension and 1st-generation DES were independent factors for the incidence of thrombus.
\end{abstract}

Conclusions: Coronary angioscopy revealed more homogeneous coverage with white neointima and less thrombus after 2nd-generation DES as compared with 1st-generation DES. These findings may explain the favorable clinical outcomes observed for patients treated with 2nd-generation DES. (Circ J 2016; 80: 1916-1921)

Key Words: Bare metal stents; Coronary angioscopy; Drug-eluting stents

$\mathbf{T}$ he drug-eluting stent (DES) has dramatically reduced the risk of restenosis and target vessel revascularization as compared with bare metal stents (BMS). ${ }^{1-3}$ Because the 1st-generation DES (1st-DES) was used widely around the world, concern was raised of an increased risk of late stent thrombosis after 1st-DES implantation., ${ }^{4,5}$ The 2ndgeneration DES (2nd-DES) has been designed with the goal of improving safety, efficacy, and device performance. Several clinical studies have demonstrated that 2nd-DES are associated with favorable clinical outcomes, including lower incidence of very late stent thrombosis, than 1st-DES. ${ }^{6-12}$

\section{Editorial p 1895}

Coronary angioscopy permits direct visualization of the intracoronary structure. It has been demonstrated that coronary angioscopy is a useful tool for estimating the neointimal coverage (NC) of stent struts, not only quantitatively by showing the coverage grade but also qualitatively by showing the plaque's yellow color (YC) grade. Several studies have compared the angioscopic findings of 2 specific types of stent, but no previous study has reported a comprehensive comparison of the

Received February 16, 2016; revised manuscript received June 5, 2016; accepted June 6, 2016; released online June 29, 2016 Time for primary review: 21 days

Department of Cardiology, Hiroshima City Hospital, Hiroshima (K.D., Y.N.); Department of Cardiology, Ehime Prefectural Imabari Hospital, Imabari (H.M., H.K.); Department of Cardiology, Okayama Red Cross Hospital, Okayama (T.S.); Department of Cardiology, Saiseikai Matsuyama Hospital, Matsuyama (K.W.); and Division of Coronary Heart Disease, Department of Internal Medicine, Hyogo College of Medicine, Nishinomiya (M.I.), Japan

Mailing address: Masaharu Ishihara, MD, PhD, FACC, Professor, Division of Coronary Heart Disease, Department of Internal Medicine, Hyogo College of Medicine, 1-1 Mukogawa-cho, Nishinomiya 663-8501, Japan. E-mail: ma-ishihara@hyo-med.ac.jp

ISSN-1346-9843 doi:10.1253/circj.CJ-16-0121

All rights are reserved to the Japanese Circulation Society. For permissions, please e-mail: cj@j-circ.or.jp 


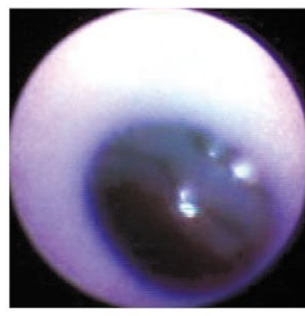

Grade 0

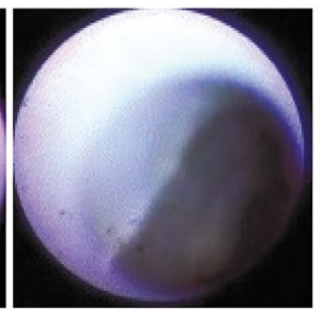

Grade 1

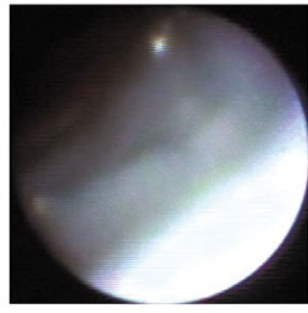

Grade 2

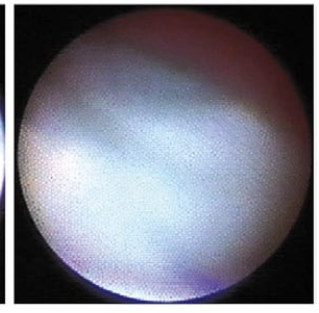

Grade 3

Figure 1. Neointimal coverage (NC) grade. Grade 0: stent struts with complete exposure (similar to immediately after implantation). Grade 1: transparent stent struts with dull light reflection; Grade 2: stent struts slightly visible, with no light reflection from stent struts; Grade 3: stent struts completely covered, and not visible through the neointima.

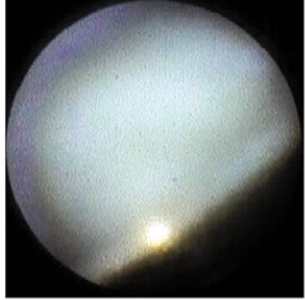

Grade 0

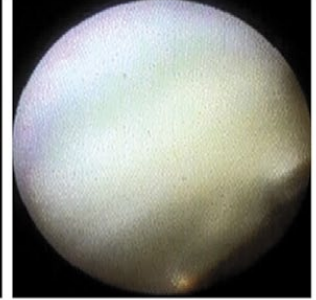

Grade 1

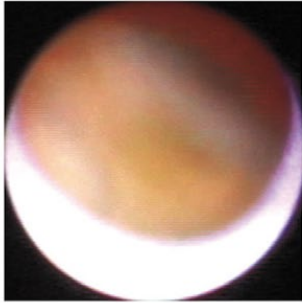

Grade 2

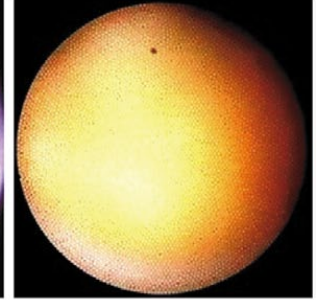

Grade 3

Figure 2. Yellow color (YC) grade of plaque: 0 (white), 1 (light yellow), 2 (yellow), and 3 (bright yellow).

chronic angioscopic findings for BMS, and 1st-DES and 2ndDES. In this study, we performed a comprehensive comparison of the coronary angioscopic findings 1 year after implantation of the 3 different stents.

\section{Methods}

\section{Study Patients}

The Multicenter Study on Intra-Coronary Angioscopy after Stent (MICASA) is a multicenter registry of coronary angioscopy conducted in 4 Japanese institutions. A total of 264 stents were observed by coronary angioscopy 1 year after percutaneous coronary intervention. There were 15 lesions treated with BMS, 90 lesions with 1st-DES (64 lesions with sirolimus-eluting stent (SES), 26 lesions with paclitaxel-eluting stent (PES)), and 159 lesions with 2nd-DES (73 lesions with everolimus-eluting stent (EES), 56 lesions with zotarolimus-eluting stent, 30 lesions with biolimus-eluting stent). When more than 1 stent were used in 1 lesion, the same type of stent was exclusively used. We excluded patients in whom we could not obtain clear angioscopic imaging because of difficulty in removing blood from the view during angioscopic examination. We did not enroll patients with in-stent restenosis.

Informed consent was given by each patient and the study protocol was reviewed and approved by the ethics committee of each hospital.

\section{Angioscopic Examination}

Catheterization was performed with a radial, brachial or femoral approach using $\geq 6 \mathrm{~F}$ catheters. After heparin administration, selective coronary angiography was performed. The coronary angioscopic examination was performed with the VFS-1300 angioscope (Nihon Kohden) and IF-783V optical fiber (Nihon Kohden). The outer section of the $4 \mathrm{~F}$ probing catheter (USCI) was used as the guide for inserting the optical fiber into the coronary artery. The angioscopic observations were made while blood was removed from the view by injection of $3 \%$ dextran-40 through the probing catheter as previously reported. ${ }^{13}$ Angioscopy images were recorded on a digital recorder.

\section{Evaluation of the Angioscopic Findings}

We assessed NC of the stent struts, YC grade of plaque and the existence of thrombus. The degree of $\mathrm{NC}$ of the stent struts was classified into 4 grades as previously described (Figure 1). ${ }^{12}$ In brief: grade $0=$ stent struts that were completely exposed (similar to immediately after implantation); grade $1=$ stent struts were visible with dull light reflection; grade $2=$ there was no light reflection from the stent struts with slightly visible struts; grade $3=$ stent struts were completely covered, and not visible through the neointima. Because most stents had $\geq 1 \mathrm{NC}$ grade, we assessed the minimum (Min-) and maximum (Max-) NC grade. The heterogeneity index was calculated as Max-NC grade minus Min-NC grade. Thrombus was defined on the basis of the criteria adopted by the European Working Group on Coronary Angioscopy. ${ }^{14}$ YC under the stent was classified into 4 grades: 0 (white), 1 (light yellow), 2 (yellow) and 3 (bright yellow) (Figure 2). The maximum YC grade of the plaques was assessed. Angioscopic evaluations were made by specialists in coronary intervention and angioscopy who were blinded to the patient's clinical status. 


\begin{tabular}{|c|c|c|c|c|c|}
\hline \multirow[b]{2}{*}{ Variable } & \multirow{2}{*}{$\begin{array}{c}\text { BMS } \\
(n=15)\end{array}$} & \multirow{2}{*}{$\begin{array}{c}\text { 1st-DES } \\
(n=90)\end{array}$} & \multirow{2}{*}{$\begin{array}{c}\text { 2nd-DES } \\
(n=159)\end{array}$} & \multicolumn{2}{|c|}{$P$ value } \\
\hline & & & & ANOVA & $\begin{array}{l}\text { 1st-DES vs. } \\
\text { 2nd-DES }\end{array}$ \\
\hline Time to angioscopy (months) & $6.9 \pm 2.0$ & $7.8 \pm 2.1$ & $10.0 \pm 2.8$ & $<0.001$ & $<0.001$ \\
\hline Age (years) & $60 \pm 9$ & $70 \pm 8$ & $69 \pm 9$ & $<0.001$ & 0.60 \\
\hline Men & $100 \%$ & $63 \%$ & $72 \%$ & 0.002 & 0.15 \\
\hline Hypertension & $60 \%$ & $63 \%$ & $69 \%$ & 0.61 & 0.40 \\
\hline Diabetes mellitus & $40 \%$ & $37 \%$ & $38 \%$ & 0.96 & 0.89 \\
\hline Dyslipidemia & $73 \%$ & $63 \%$ & $64 \%$ & 0.74 & 0.89 \\
\hline Current smoking & $7 \%$ & $19 \%$ & $10 \%$ & 0.11 & 0.07 \\
\hline Lesion location & & & & 0.48 & 0.21 \\
\hline LAD & $40 \%$ & $61 \%$ & $52 \%$ & & \\
\hline LCX & $20 \%$ & $9 \%$ & $15 \%$ & & \\
\hline $\mathrm{RCA}$ & $40 \%$ & $30 \%$ & $33 \%$ & & \\
\hline ACS & $67 \%$ & $38 \%$ & $38 \%$ & 0.10 & $>0.99$ \\
\hline No. of stents per lesion & $1.0 \pm 0.0$ & $1.2 \pm 0.4$ & $1.2 \pm 0.4$ & 0.26 & 0.69 \\
\hline Stent diameter $(\mathrm{mm})$ & $3.6 \pm 0.4$ & $3.1 \pm 0.3$ & $3.0 \pm 0.3$ & $<0.001$ & 0.69 \\
\hline Stent length (mm) & $17 \pm 4$ & $24 \pm 11$ & $23 \pm 10$ & 0.02 & 0.17 \\
\hline \multicolumn{6}{|l|}{ Medications at follow-up } \\
\hline DAPT & $53 \%$ & $98 \%$ & $88 \%$ & $<0.001$ & 0.056 \\
\hline Statin & $87 \%$ & $79 \%$ & $88 \%$ & 0.38 & 0.13 \\
\hline LDL-C (mg/dl) & $95 \pm 30$ & $94 \pm 32$ & $84 \pm 23$ & 0.02 & 0.009 \\
\hline eGFR $\left(\mathrm{ml} / \mathrm{min} / 1.73 \mathrm{~m}^{2}\right)$ & $70 \pm 15$ & $61 \pm 14$ & $63 \pm 18$ & 0.23 & 0.57 \\
\hline $\mathrm{HbA} 1 \mathrm{c}(\%)$ & $6.3 \pm 0.8$ & $6.1 \pm 0.8$ & $6.3 \pm 1.0$ & 0.61 & 0.33 \\
\hline
\end{tabular}

1st, 1st-generation; 2nd, 2nd-generation; ACS, acute coronary syndrome; BMS, bare metal stent; DAPT, dual antiplatelet therapy; DES, drug-eluting stent; LAD, left anterior descending artery; LCX, left circumflex artery; $\mathrm{RCA}$, right coronary artery.
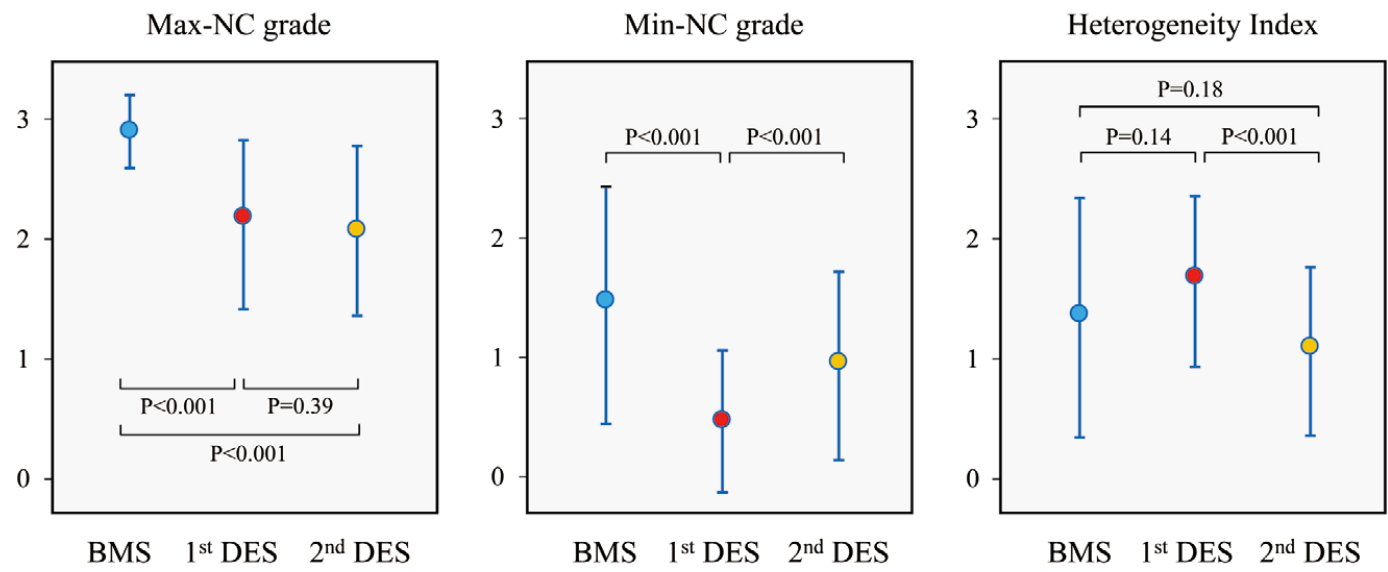

Figure 3. Min-NC grade, Max-NC grade and heterogeneity index. Max-NC and Min-NC grades were significantly higher with 1st-generation DES (1st-DES) and 2nd-generation DES (2nd DES) than with BMS. Although there was no significant difference in Max-NC grade, the Min-NC grade is significantly higher for 2nd-DES than for 1st-DES. The heterogeneity index was significantly smaller for 2nd-DES than for 1st-DES, and comparable to BMS. BMS, bare metal stent; DES, drug-eluting stent; NC, neointimal coverage.

\section{Statistical Analysis}

Continuous and ordinal variables are shown as mean \pm SD. Student's t-test and ANOVA were used for continuous variables. Wilcoxon test was used for ordinal variables. Categorical variables were expressed as frequencies and analyzed by chi-square statistics or Fisher's exact test. Logistic regression analysis was performed to identify independent predictors of yellow plaque and thrombus. Yellow plaque was defined as YC grade 2 or 3 . We used the JMP statistical package (version 5.0.1 J, SAS Institute, Cary, NC, USA) for all statistical tests. A significance level of 0.05 was used and 2-tailed tests were applied. 

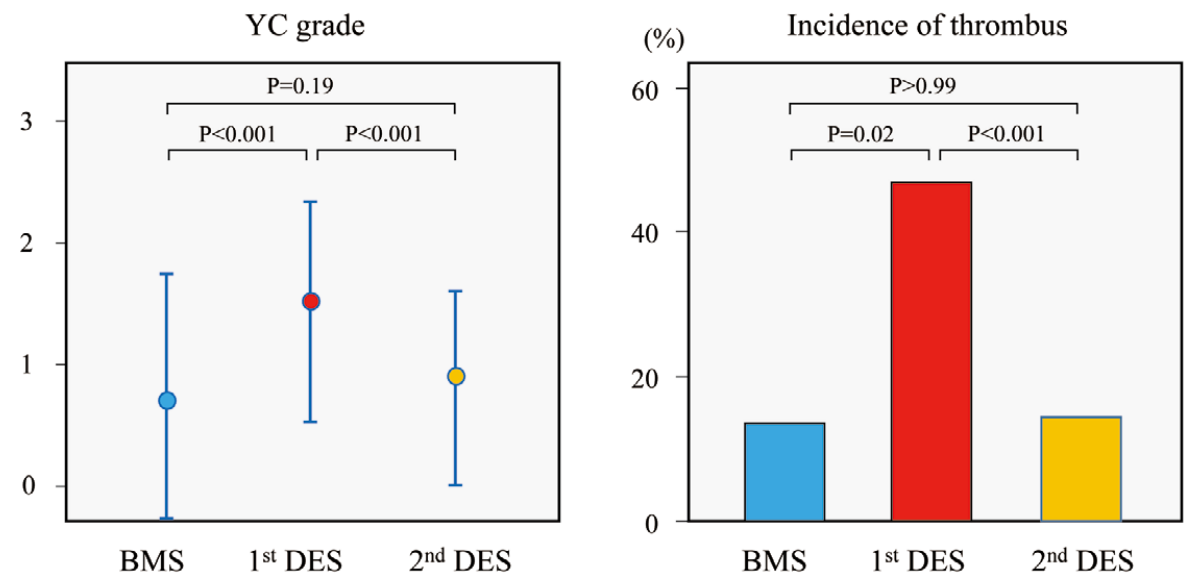

Figure 4. Yellow color (YC) grade of plaque and incidence of thrombus. YC grade was significantly higher with 1st-generation DES (1st-DES) than with BMS or 2nd-generation DES (2nd-DES). There was no significant difference in YC grade between BMS and 2 nd-DES. The incidence of thrombus was lower with 2nd-DES than with 1st-DES, and was similar to BMS. BMS, bare metal stent; DES, drug-eluting stent.

\section{Baseline Characteristics}

\section{Results}

The baseline clinical characteristics of each group are shown in the Table. Duration from stent implantation to coronary angioscopy of BMS, 1st-DES and 2nd-DES was $6.9 \pm 2.0$ months, 7.8 2 2.1 months, and 10.0 \pm 2.8 months, respectively. Use of DES was associated with higher age, more females, smaller stent diameter, longer stent length, and dual antiplatelet therapy at the time of angioscopy.

\section{Angioscopic Findings}

The angioscopic findings of NC are shown in Figure 3. Max$\mathrm{NC}$ and Min-NC grades were significantly lower for 1st-DES (2.2 $\pm 0.7, \mathrm{P}<0.001$ for Max-NC; $0.5 \pm 0.6, \mathrm{P}<0.001$ for Min-NC) and 2 nd-DES $(2.1 \pm 0.7, \mathrm{P}<0.001$ for Max-NC; $1.0 \pm 0.8, \mathrm{P}=0.01$ for Min-NC) than for BMS (2.9 \pm 0.3 and $1.5 \pm 1.0$, respectively). Although there was no significant difference in Max-NC grade, the Min-NC grade was significantly higher for 2nd-DES than for $1 \mathrm{st}-\mathrm{DES}$. The heterogeneity index was significantly smaller for 2nd-DES $(1.1 \pm 0.7)$ than for 1 st-DES $(1.7 \pm 0.7, \mathrm{P}<0.001)$, and comparable to that for BMS (1.4 \pm 1.0$)$.

YC grade and incidence of thrombus are shown in Figure 4. YC grade was significantly higher with 1 st-DES (1.5 \pm 0.9$)$ than with BMS $(0.7 \pm 1.0, \mathrm{P}=0.001)$ and 2nd-DES $(0.9 \pm 0.8$, $\mathrm{P}<0.001)$. There was no significant difference in $\mathrm{YC}$ grade between BMS and 2nd-DES. Higher YC grade of the plaque was associated with higher low-density lipoprotein (LDL) level $(\mathrm{P}=0.0007)$, higher frequency of acute coronary syndrome (ACS) diagnosed at initial percutaneous coronary intervention $(\mathrm{P}=0.008)$, 1st-DES $(\mathrm{P}<00001)$, lower Min-NC grade $(\mathrm{P}<0.0001)$, lower Max-NC grade $(\mathrm{P}=0.0006)$, lower heterogeneity index $(\mathrm{P}=0.003)$, and higher incidence of thrombus $(\mathrm{P}=0.0001)$. The incidence of thrombus was lower with 2 ndDES (15\%) than with 1st-DES $(47 \%, \mathrm{P}<0.001)$, and was similar to that for BMS (13\%, P>0.99) (Figure 4). Presence of thrombus was associated with lack of hypertension $(55 \%$ vs. $70 \%, \mathrm{P}=0.026)$, shorter time to follow-up angioscopy (8.3 \pm 2.5 vs. $9.2 \pm 2.8, \mathrm{P}=0.02)$, lower Min-NC grade $(0.5 \pm 0.6$ vs. $1.0 \pm 0.8, \mathrm{P}<0.001)$, larger heterogeneity index $(1.7 \pm 0.8$ vs. $1.2 \pm 0.7, \mathrm{P}<0.001)$, higher $\mathrm{YC}$ grade $(1.5 \pm 0.8$ vs. $1.0 \pm 0.9$, $\mathrm{P}<0.001)$ and use of 1 st-DES $(61 \%$ vs. $25 \%, \mathrm{P}<0.001)$. When patients were divided into ACS or stable angina pectoris (SAP) groups, similar findings were observed in both (Figures S1,S2). Multivariate analysis showed that LDL $(\mathrm{P}=0.01)$, 1st-DES $(\mathrm{P}<0.0001)$, and ACS $(\mathrm{P}=0.006)$ were independent factors for yellow plaque, and that hypertension $(\mathrm{P}=0.04)$ and 1 st-DES $(\mathrm{P}=0.002)$ were independent factors for thrombus.

\section{Discussion}

In this study, we compared the chronic angioscopic findings of BMS, 1st-DES and 2nd-DES. The main findings of this study were: (1) Max-NC and Min-NC grades with 1st- and 2nd-DES were lower than with BMS, (2) the Max-NC grade with 2ndDES was similar to that with 1st-DES, but the Min-NC grade with 2nd-DES was higher, and (3) the heterogeneous index, YC grade and incidence of thrombus with 2nd-DES were all lower than with 1st-DES and were comparable to BMS.

Since their introduction in the early 2000s, DES have been widely used because of the dramatic decrease in in-stent restenosis and the need for target lesion revascularization. Instead, late stent failure, which includes late stent thrombosis and late catch-up phenomenon, were raised as a critical matter. Oversuppression of neointimal proliferation by the drugs and hypersensitivity to the polymers are thought to be responsible for late stent failure. The 2 nd-DES were developed to overcome these disadvantages of 1st-DES, and have shown favorable clinical outcomes. In a meta-analysis of 51 randomized trials with $\geq 3$ years' follow-up of more than 50,000 patients, Palmerini et al demonstrated that 2 nd-DES have substantially improved long-term safety and efficacy outcomes compared with 1stDES. ${ }^{15}$

The underlying mechanisms for late stent failure after DES have been investigated histologically and by intravascular imaging modalities. ${ }^{16}$ From the results of 81 autopsies of patients with 1st-DES, Finn et al showed that the ratio of uncovered to total stent struts and heterogeneity of healing 
were predictors of late stent thrombosis. ${ }^{17}$ They also reported that neoatherosclerosis is a frequent finding with DES and occurs earlier than with BMS, suggesting another factor contributing to late stent failure. ${ }^{18}$ Intravascular imaging has been used to evaluate vascular responses to stents in living patients. Optical coherence tomography (OCT), as well as coronary angioscopy, is a useful imaging modality, because it has a 10-fold higher resolution than intravascular ultrasound. OCT permits qualitative assessment of $\mathrm{NC}$, whereas the coverage grade by angioscopy is semiquantitative. However, angioscopy is the gold standard for detecting yellow plaque by full-color visualization and is superior to OCT for detecting intracoronary thrombus. Inoue et al compared the OCT and angioscopy findings for 26 coronary lesions at 10 months after SES implantation. They reported that the OCT-based definition of thrombus may underestimate the presence of subclinical thrombus detected by angioscopy. ${ }^{19}$

In the current study using coronary angioscopy, we showed that the Min-NC grade, heterogeneity index and YC grade were associated with thrombus. A recent prospective study has demonstrated that yellow plaque at the lesion previously treated with DES predicts future late stent failure. ${ }^{20}$ Hypertension was negatively associated with thrombus, which was an unexpected finding, because most of the previous studies reported no association between hypertension and stent thrombosis. However, it is noteworthy that use of 1st-DES had the strongest association with thrombus.

Coronary angioscopy has been used to compare the vascular responses to different types of stent. Awata et al performed serial coronary angioscopic examinations after BMS and SES implantation. ${ }^{21}$ SES demonstrated lower NC grade, more intense yellow plaque and higher incidence of thrombus, while the BMS were completely covered by white neointima by 3-6 months. In another angioscopic study, PES showed the most heterogeneous neointimal formation and the highest incidence of thrombus formation compared with SES and BMS.22 With respect to 2nd-DES, we have previously reported that EES was associated with more homogeneous NC coverage and less YC grade than SES. ${ }^{23}$ These studies were, however, single-center studies with a sample size $<100$ patients. Using OCT, Choi et al reported that EES might have more favorable vascular responses after stent implantation than SES. ${ }^{24}$

The current study is the first multicenter registry to perform a comprehensive comparison of the coronary angioscopic findings for BMS, 1st-DES and 2nd-DES. 1st-DES and 2ndDES showed similar Max-NC grade, and a corresponding equivalent low incidence of in-stent restenosis. Min-NC was higher, and heterogeneous index and YC grade were lower with 2nd-DES than with 1st-DES, suggesting a relation to the lower incidence of thrombus with 2 nd-DES. These angioscopic findings endorse the clinical efficacy of 2nd-DES similar to that for 1st-DES, and safety comparable to that of BMS.

\section{Study Limitations}

This was an observational study. Selection of stent depended on physicians and the baseline characteristics of each group were not matched. Reflecting market penetration, the number of BMS was small as compared with the number of DES. However, all consecutive patients undergoing coronary angioscopy of stent-implanted lesions were included. This registry enrolled only coronary angioscopic examination 1 year after stent implantation, and serial changes in the angioscopic findings after stenting were not assessed. Because of the insurance regulations of Japan, other imaging modalities, including OCT and intravascular ultrasound, were not routinely used simulta- neously. Finally, lack of a core laboratory was another limitation of this multicenter study. However, for the past 10 years, the MICASA group has held regular conferences (Chugokushikoku angioscopy conference) twice each year to reach consensus on coronary angiographic findings.

\section{Conclusions}

Coronary angioscopy revealed more homogeneous coverage with white neointima and less thrombus after 2nd-DES as compared with 1 st-DES. These angioscopic findings endorse the clinical efficacy of 2nd-DES as similar to that of 1st-DES and a safety profile comparable to that of BMS. These findings may explain the favorable clinical outcomes of patients treated with 2nd-DES.

\section{Acknowledgments}

This study was supported by JSPS KAKENHI Grant Number 26461101.

\section{Disclosures}

M.I. received lecture fees from MSD K.K., Bayer Yakuhin, Ltd, Daiichi Sankyo Co, Ltd, Abbott Vascular Japan Co, Ltd, Sanofi K.K., Kissei Pharmaceutical Co, Ltd and research funding from Abbott Vascular Japan Co, Ltd, Boston Scientific Japan K.K., Sanofi K.K. MSD K.K., Bayer Yakuhin, Ltd, Daiichi Sankyo Co, Ltd, Goodman Co, Ltd, and MID, Inc.

\section{References}

1. Moses JW, Leon MB, Popma JJ, Fitzgerald PJ, Homes DR, O'Shaughnessy C, et al; for the SIRIUS Investigators. Sirolimuseluting stents versus standard stents in patients with stenosis in a native coronary artery. $N$ Engl J Med 2003; 349: 1315-1323.

2. Morice MC, Serruys PW, Sousa JE, Fajadet J, Hayashi EB, Perin M, et al; for the RAVEL Study Group. A randomized comparison of a sirolimus-eluting stent with a standard stent for coronary revascularization. $N$ Engl J Med 2002; 346: 1773-1780.

3. Kyung WP, Si HK, Woo YC, Hae YL, Jin SP, Hyun JK, et al. 'Real World' comparison of drug-eluting stents vs bare metal stents in the treatment of unselected patients with acute ST-segment elevation myocardial infarction. Circ J 2010; 74: $1111-1120$.

4. Daemen J, Wenaweser P, Tsuchida K, Abrecht L, Vaina S, Morger $\mathrm{C}$, et al. Early and late coronary thrombosis of sirolimus-eluting and paclitaxel-eluting stents in routine clinical practice: Data from a large two-institutional cohort study. Lancet 2007; 369: 667-678.

5. McFadden EP, Stabile E, Regar E, Cheneau E, Ong AT, Kinnaird T, et al. Late thrombosis in drug-eluting coronary stents after discontinuation of antiplatelet therapy. Lancet 2004; 364: 1519-1521.

6. Baber U, Mehran R, Sharma SK, Brar S, Yu J, Suh JW, et al. Impact of the everolimus-eluting stent on stent thrombosis: A meta-analysis of 13 randomized trials. J Am Coll Cardiol 2011; 58: 1569-1577.

7. Palmerini T, Biondi-Zoccai G, Della Riva D, Stettler C, Sangiorgi D, D'Ascenzo F, et al. Stent thrombosis with drug-eluting and baremetal stents: Evidence from comprehensive network meta-analysis. Lancet 2012; 379: $1393-1402$.

8. Stefanini GG, Byrne RA, Serruys PW, Waha AD, Meier B, Massberg $\mathrm{S}$, et al. Biodegradable polymer drug-eluting stents reduce the risk of stent thrombosis at 4 years in patients undergoing percutaneous coronary intervention: A pooled analysis of individual patient data from the ISAR-TEST 3, ISAR-TEST 4, and LEADERS randomized trials. Eur Heart J 2012; 33: 1214-1222.

9. Waha AD, Cassese S, Park DW, Burzotta F, Byrne RA, Tada T, et al. Everolimus-eluting versus sirolimus-eluting stents: An updated meta-analysis of randomized trials. Clin Res Cardiol 2012; 101: $461-467$.

10. Raber L, Margo M, Stefanini GG, Kalesan B, Domburg RT, Onuma $\mathrm{Y}$, et al. Very late coronary stent thrombosis of a newer-generation everolimus-eluting stent compared with early-generation drug-eluting stents: A prospective cohort study. Circulation 2012; 125: 1110-1121.

11. Tada T, Byrne RA, Simunovic I, King LA, Cassesse S, Joner M, et al. Risk of stent thrombosis among bare-metal stents, first-generation drug-eluting stents, and second-generation drug-eluting stents. J Am Coll Cardiol Interv 2013; 6: 1267-1274.

12. Akazawa Y, Matsuo K, Ueda Y, Nishio M, Hirata A, Asai M, et al. Atherosclerotic change at one year after implantation of Endeavor zotarolimus-eluting stent vs. everolimus-eluting stent. Circ J 2014; 
78: $1428-1436$.

13. Dai K, Ishihara M, Inoue I, Kawagoe T, Shimatani Y, Kurisu S, et al. Coronary angioscopic findings eight months after sirolimus-eluting stent implantation: A comparison between ST-elevation myocardial infarction and stable angina pectoris. Euro Intervention 2010; 6: $251-256$.

14. Heifer PD, Foley DP, Hillege HL, Lablanche JM, Dijk RBV, Franzen $\mathrm{D}$, et al. The "Ermenonville" classification of observations at coronary angioscopy: Evaluation of intra- and inter-observer agreement. Eur Heart J 1994; 15: 815-822.

15. Palmerini T, Benedetto U, Zoccai GB, Riva DD, Reggiani LB, Smits PC, et al. Long-term safety of drug-eluting and bare-metal stents. $J$ Am Coll Cardiol 2015; 65: 2496-2507.

16. Otsuka F, Vorpahl M, Nakano M, Foerst J, Newell JB, Sakakura K, et al. Pathology of second-generation everolimus-eluting stents versus first-generation sirolimus- and paclitaxel-eluting stents in humans. Circulation 2014; 129: $211-223$.

17. Finn AV, Joner M, Nakazawa G, Kolodgie F, Newell J, John MC, et al. Pathological correlates of late drug-eluting stent thrombosis: Strut coverage as a marker of endothelialization. Circulation 2007; 115: $2435-2441$

18. Nakazawa G, Otsuka F, Nakano M, Vorpahl M, Yazdani SK, Ladich $\mathrm{E}$, et al. The pathology of neoatherosclerosis in human coronary implants. J Am Coll Cardiol 2011; 57: 1314-1322.

19. Inoue T, Shinke T, Otake H, Nakagawa M, Hariki H, Osue T, et al Neoatherosclerosis and mural thrombus detection after sirolimuseluting stent implantation: Comparison of angioscopy and optical coherence tomography assessment for color-based tissue characterization. Circ J 2014; 78: $92-100$.

20. Ueda Y, Matsuo K, Nishimoto Y, Sugihara R, Hirata A, Nemoto T, et al. In-stent yellow plaque at 1 year after implantation is associated with future event of very late stent failure. J Am Coll Cardiol Interv
2015; 8: 814-821.

21. Awata M, Kotani J, Uematsu M, Morozumi T, Watanabe T, Onishi $\mathrm{T}$, et al. Serial angioscopic evidence of incomplete neointimal coverage after sirolimus-eluting stent implantation: Comparison with baremetal stents. Circulation 2007; 116: 910-916.

22. Hara M, Nishino M, Taniike M, Makino N, Kato H, Egami Y, et al Difference of neointimal formational pattern and incidence of thrombus formation among 3 kinds of stents: An angioscopic study. $J$ Am Coll Cardiol Interv 2010; 3: 215-220.

23. Dai K, Ishihara M, Inoue I, Kawagoe T, Shimatani Y, Miura F, et al Coronary angioscopic findings 9 months after everolimus-eluting stent implantation compared with sirolimus-eluting stents. $J$ Cardiol 2013; 61: 22-30.

24. Choi HH, Kim JS, Yoon DH, Hong KS, Kim TH, Kim BK, et al Favorable neointima coverage in everolimus-eluting stent at 9 months after stent implantation: Comparison with sirolimus-eluting stent using optical coherence tomography. Int J Cardiovasc Imaging 2012; 28: 491-497.

\section{Supplementary Files}

Supplementary File 1

Figure S1. Min-NC grade, Max-NC grade, heterogeneity index, YC grade and incidence of thrombus in stable angina pectoris (SAP) patients.

Figure S2. Min-NC grade, Max-NC grade, heterogeneity index, YC grade and incidence of thrombus in acute coronary syndrome (ACS) patients.

Please find supplementary file(s);

http://dx.doi.org/10.1253/circj.CJ-16-0121 